\title{
EXCHANGEABLE EVENTS AND COMPLETELY MONOTONIC SEQUENCES
}

\author{
CLARK H. KIMBERLING ${ }^{1}$
}

1. Introduction. We investigate the probability theory of an infinite sequence of events, all having the same probability, and we assume the events are exchangeable. That is, for each positive integer $n$, each pair of $n$-fold intersections of events have the same probability. In the familiar game of tossing a coin forever, the events "heads" provide an example of a sequence of exchangeable events. A less trivial example is provided by Pólya's urn model. (See, for example, Feller [1, p. 226] .)

We show in Theorem 1 that a sequence of events of common probability is exchangeable if and only if the sequence of real numbers whose $n$th term is the probability common to the $n$-fold intersections of events is a completely monotonic sequence. Theorem 2 asserts that for such events, Kolmogorov's Strong Law of Large Numbers holds if and only if the events are independent. Theorems 3, 4, 5, and 6 describe probabilities of unions and intersections of exchangeable events of common probability.

We recall now preliminaries from Feller [1, p. 225], Widder [4, p. 108 and p. 12], and Hardy [2, pp. 279-282]. For a sequence $\mu_{0}, \mu_{1}, \cdots$ of real numbers, we denote by $\Delta^{m} \mu_{q}$ the sum

$$
\sum_{k=0}^{m}(-1)^{k}\left(\begin{array}{c}
m \\
k
\end{array}\right) \mu_{q+m-k}
$$

and define $\mu_{0}, \mu_{1}, \cdots$ to be completely monotonic if

$$
(-1)^{m} \Delta^{m} \mu_{q} \geqq 0 \quad \text { for } m, q=0,1, \cdots,
$$

and minimally completely monotonic if for every $\mu<\mu_{0}$ the sequence $\boldsymbol{\mu}, \boldsymbol{\mu}_{1}, \mu_{2}, \cdots$ is not completely monotonic.

A fundamental theorem of Hausdorff is that $\mu_{0}, \mu_{1}, \cdots$ is completely monotonic if and only if there exists a bounded nondecreasing function $\alpha$ from $[0,1]$ into $[0, \infty)$ such that

Received by the editors June 28, 1971 and, in revised form, March 20, 1972.

AMS (MOS) subject classifications (1970). Primary 39A05, 60G05, 40-01; Secondary $26 \mathrm{~A} 48,60-01$.

${ }^{1}$ The author is grateful for support from the University of Evansville and from NSF Grant GY5595. 


$$
\mu_{n}=\int_{0}^{1} t^{n} d \alpha(t) \text { for } n=0,1, \cdots
$$

We always take $\alpha$ to be the unique normalized function of Widder [4, p. 100] and refer to $\alpha$ as the distributor for $\mu_{0}, \mu_{1}, \cdots$.

The essentials of the proof of the next theorem, referred to in the sequel as Hardy's theorem, may be found in Hardy [2] and Widder [4]. Suppose $\mu_{0}, \mu_{1}, \cdots$ is a completely monotonic sequence with distributor $\alpha$. Then the following are equivalent:

(i) There exists $\mu$ such that the sequence $\mu, \mu_{0}, \mu_{1}, \cdots$ is completely monotonic.

(ii) $\sum_{i=0}^{\infty}(-1)^{i} \Delta^{i} \mu_{0}<\infty$.

(iii) There exists a distributor $\gamma$ such that

$$
\alpha(t)=\int_{0}^{t} s d \gamma(s)
$$

in this case, $\boldsymbol{\gamma}$ is the distributor of $\mu, \mu_{0}, \mu_{1}, \cdots$.

(iv) $\int_{0}^{1}(1 / t) d \alpha(t)<\infty$.

That $\mu$ for which $\mu, \mu_{0}, \mu_{1}, \cdots$ is minimally completely monotonic, when such a $\mu$ exists, is

$$
\sum_{i=0}^{\infty}(-1)^{i} \Delta^{i} \mu_{0}=\int_{0}^{1} \frac{1}{t} d \alpha(t)
$$

2. Main results. We define a sequence of real numbers $\mu_{0}, \mu_{1}, \mu_{2}, \cdots$, where $\mu_{0}=1$, to be admissible if there exists a probability space $(\Omega, A, P)$ and events $A_{n}$ in $A$ such that

$$
P\left(A_{n_{1}} \cdots A_{n_{m}}\right)=\mu_{m}
$$

whenever

$$
1 \leqq n_{1}<\cdots<n_{m} \text { for } m=1,2, \cdots
$$

We say that the events $A_{n}$ and their indicators $I_{n}$ are exchangeable with respect to $\mu_{0}, \mu_{1}, \cdots$.

Theorem 1. Let $\mu_{0}, \mu_{1}, \cdots$ be a sequence with $\mu_{0}=1$. Then the sequence $\mu_{0}, \mu_{1}, \cdots$ is admissible if and only if it is completely monotonic.

Proof. Granted admissibility, complete monotonicity is immediate by de Finetti's theorem (see, for example, Feller [1, p. 225]).

To prove the converse, we define for $m=1,2, \cdots$ an $m$-place function $F_{1, \cdots, m}$ by 


$$
F_{1, \cdots, m}\left(x_{1}, \cdots, x_{m}\right)=\left\{\begin{aligned}
0 & \text { if } \min \left\{x_{1}, \cdots, x_{m}\right\}<0, \\
\mu_{m-j} & \text { if } \min \left\{x_{1}, \cdots, x_{m}\right\} \geqq 0, \\
& \text { if exactly } m-j \text { of these } \\
& \text { coordinates are in }[0,1) \text { and } \\
& j \text { coordinates are } \geqq 1 .
\end{aligned}\right.
$$

For each finite collection $n_{1}<\cdots<n_{k}$ of indices, define $F_{n_{1}, \cdots, n_{k}}$ marginally, viz.,

$$
F_{n_{1}, \cdots, n_{k}}\left(x_{1}, \cdots, x_{k}\right)=F_{1,2, \cdots, n_{k}}\left(1, \cdots, 1, x_{1}, 1, \cdots, \cdots, 1, x_{k}\right),
$$

where $x_{j}$ occupies the $n_{j}$ th place for $j=1, \cdots, k$. For each permutation $\lambda_{n_{1}}, \cdots, \lambda_{n_{k}}$ of $n_{1}, \cdots, n_{k}$, define

$$
F_{\lambda_{n_{1}}, \cdots, \lambda_{n_{k}}}=F_{n_{1}, \cdots, n_{k}} \text {. }
$$

Then

$$
F_{\lambda_{1}, \cdots, \lambda_{k}}=F_{1}, \cdots,,_{k}
$$

for every set of $k$ distinct indices $\lambda_{1}, \cdots, \lambda_{k}$.

The collection

$$
\mathscr{F}=\left\{F_{\lambda_{1}}, \cdots, \lambda_{n}:\left\{\lambda_{1}, \cdots, \lambda_{n}\right\} \text { is a finite collection of positive integers }\right\}
$$

clearly satisfies items a, b, c, e, and f of the hypothesis of the Kolmogorov theorem, as presented in Tucker [3, p. 30]. We proceed with an inductive argument to show that item $\mathrm{d}$ is also satisfied. Letting $\boldsymbol{\mu}_{F_{1}, \cdots, m}$ be the probability measure induced on $\boldsymbol{R}^{m}$ by $F_{1, \cdots, m}$, we wish to show that for every $m$ dimensional cell $(a, b]=\left(\left(a_{1}, \cdots, a_{m}\right)\right.$, $\left.\left(b_{1}, \cdots, b_{m}\right)\right]$, we have

$$
\mu_{F_{1}, \cdots, m}(a ; b]=\sum_{k=0}^{m}(-1)^{k} \sum_{\delta \in \Delta_{k, m}} F_{1, \cdots, m}(\delta) \geqq 0,
$$

where $\delta$ ranges through the set $\Delta_{k, m}$ of $\left(\begin{array}{c}m \\ k\end{array}\right)$ vertices of $(a, b]$ which consist of $k a_{i}$ 's and $m-k b_{i}$ 's, for $m=1,2, \cdots$.

For $m=1,(1)$ is just $F_{1}(b)-F_{1}(a) \geqq 0$. Now fix $m>1$, and suppose that $(1)$ holds in $\mathscr{F}$ for all cells $(a, b]$ of dimension $m-1$. For $q=m$, we shall write just $F$ for $F_{1, \cdots, q}$. Let

$$
(a, b]=\left(\left(a_{1}, \cdots, a_{m}\right),\left(b_{1}, \cdots, b_{m}\right)\right]
$$

be an arbitrary $m$ dimensional cell. 
Lemma. Suppose that for some $j$ satisfying $1 \leqq j \leqq m$, one of th following is true:

$$
a_{j} \leqq b_{j}<0, \quad 0 \leqq a_{j} \leqq b_{j}<1, \quad \text { or } \quad 1 \leqq a_{j} \leqq b_{j}
$$

Then we see that (1) holds with equality, since the terms in the sun in (1) can be paired as

$$
F\left(x_{1}, \cdots, b_{j}, \cdots, x_{m}\right)-F\left(x_{1}, \cdots, a_{j}, \cdots, x_{m}\right),
$$

and each such difference is equal to zero.

Now let $q$ be the number of components $b_{i}$ of $b$ such that $b_{i}<1$ If the hypothesis of the lemma does not hold, we have $a_{i}<0$ for thos $i$ satisfying $b_{i}<1$.

Case 1 . If the remaining $m-q a_{i}$ 's satisfy $0 \leqq a_{i}<1$, then

$$
F(\delta)=\left\{\begin{array}{cl}
\mu_{q} . & \text { if } \delta=b, \\
\cdot & \\
\mu_{q+k} & \text { if } \delta \text { has exactly } k \text { nonnegative } a_{i}^{\prime} s \\
\cdot & \\
\mu_{m} & \text { if } \delta \text { has } m-q \text { nonnegative } a_{i} \text { 's, } \\
0 & \begin{array}{l}
\text { otherwise, since in all the remaining } \\
\text { cases, at least one component of } \delta \\
\text { is negative. }
\end{array}
\end{array}\right.
$$

If $0 \leqq k \leqq m-q$, then $\left({ }^{m} \bar{k}^{q}\right)$ is the number of vertices having exactly $k$ nonnegative $a_{i}$ 's, so that

Thus,

$$
\sum_{\delta \in \Delta_{k, m}} F(\delta)=\left(\begin{array}{c}
m-q \\
k
\end{array}\right) \mu_{q+k} .
$$

$$
\begin{aligned}
\sum_{k=0}^{m}(-1)^{k} \sum_{\delta^{\prime} \in \Delta_{k, m}} F_{1, \cdots, m}(\delta) & =\sum_{k=0}^{m-q}(-1)^{k} \sum_{\delta \in \Delta_{k, m}} F_{1, \cdots, m}(\boldsymbol{\delta}) \\
& =\sum_{k=0}^{m-q}(-1)^{k}\left(\begin{array}{c}
m-q \\
k
\end{array}\right) \mu_{q+k} \\
& =(-1)^{m-q} \sum_{k=0}^{m-q}(-1)^{k}\left(\begin{array}{c}
m-q \\
k
\end{array}\right) \mu_{m-k} \\
& =(-1)^{m-q} \Delta^{m-q} \mu_{q} \geqq 0 .
\end{aligned}
$$


Case 2. If the lemma does not apply and if for some $k$ satisfying $1 \leqq k \leqq m$, we have $b_{k} \geqq 1$ and $a_{k}<0$, then we define

$$
a^{\prime}=\left(a_{1}, \cdots, a_{k-1}, a_{k+1}, \cdots, a_{m}\right)
$$

and

$$
b^{\prime}=\left(b_{1}, \cdots, b_{k-1}, b_{k+1}, \cdots, b_{m}\right)
$$

and claim that

$$
\mu_{F}(a, b]=\mu_{F_{1}, \cdots, m-1}\left(a^{\prime}, b^{\prime}\right],
$$

this second expression being nonnegative by the induction hypothesis. To see that

$$
\mu_{F}(a, b]=\mu_{F_{1, \cdots, m-1}}\left(a^{\prime}, b^{\prime}\right],
$$

define for each vertex $\delta$ of $(a, b]$ the vertex $\delta^{\prime}$ of $\left(a^{\prime}, b^{\prime}\right]$ formed by removal of the $k$ th component of $\delta$. Then

$$
F(\delta)= \begin{cases}F_{1, \cdots, m-1}\left(\delta^{\prime}\right) & \text { if the } k \text { th component of } \delta \text { is } b_{k}, \\ 0 & \text { if the } k \text { th component of } \delta \text { is } a_{k} .\end{cases}
$$

Now decomposing $\Delta_{k, m}$ into

$$
\Delta(b)=\left\{\delta \in \Delta_{k, m}: b_{k} \text { is the } k \text { th component of } \delta\right\}
$$

and

$$
\Delta(a)=\left\{\delta \in \Delta_{k, m}: a_{k} \text { is the } k \text { th component of } \delta\right\}
$$

we have

$$
\sum_{\delta \in \Delta_{k, m}} F(\delta)=\sum_{\delta \in \Delta(b)} F(\delta)+\sum_{\delta \in \Delta(a)} F(\delta)=\sum_{\delta^{\prime} \in \Delta_{k, m-1}} F_{1, \cdots, m-1}\left(\delta^{\prime}\right),
$$

so that

$$
\begin{aligned}
\sum_{k=1}^{m}(-1)^{k} \sum_{\delta \in \Delta_{k, m}} F(\delta) & =\sum_{k=1}^{m-1}(-1)^{k} \sum_{\delta \in \Delta_{k, m-1}} F_{1, \cdots, m-1}\left(\delta^{\prime}\right) \\
& =\mu_{F_{1}, \cdots, m-1}\left(a^{\prime}, b^{\prime}\right] .
\end{aligned}
$$

This completes a proof that item d of the hypothesis of the Kolmogorov theorem is satisfied by the collection $\mathfrak{\mho}$. Applying the Kolmogorov theorem, we now conclude that there exist a probability space $(\Omega, A, P)$ and random variables $\xi_{n}$ over $\Omega$ whose distribution functions 
and joint distribution functions are, with corresponding indices, those in $\mathscr{F}$. In particular

$$
F_{\xi_{n}}(x)= \begin{cases}0 & \text { if } x<0 \\ \mu_{1} & \text { if } 0 \leqq x<1 \\ 1 & \text { if } x \geqq 1\end{cases}
$$

and letting $A_{n}=\xi_{n}^{-1}(-\infty, 0]$, we have for every positive integer $m$, and indices $n_{1}<\cdots<n_{m}$,

$$
\begin{aligned}
P\left(\bigcap_{j=1}^{m} A_{n_{j}}\right) & =P\left(\bigcap_{j=1}^{m} \xi_{n_{j}}^{-1}(-\infty, 0]\right) \\
& =F_{n_{1}, \cdots, n_{m}}(0, \cdots, 0)=F_{1, \cdots, m}(0, \cdots, 0)=\mu_{m} .
\end{aligned}
$$

Therefore, the sequence $\mu_{0}, \mu_{1}, \cdots$ is admissible.

TheOREM 2. Let $\mu_{0}, \mu_{1}, \cdots$ be a sequence admissible with respect to indicators $I_{1}, I_{2}, \cdots$. Then there exists a constant random variable c such that the sequence

$$
I_{1}, \frac{1}{2}\left(I_{1}+I_{2}\right), \cdots,\left(I_{1}+\cdots+I_{n}\right) / n, \cdots
$$

of arithmetic means converges in probability to $c$ if and only if the indicators $I_{1}, I_{2}, \cdots$ are independent, in which case the random variable $c$ is given by

$$
c(\omega)=\mu_{1} \text { for all } \omega \in \Omega .
$$

Proof. It is well known (for example, Tucker [3, pp. 123-124]) that if the $I_{n}$ are independent, then the arithmetic means converge not only in probability to $\mu_{1}$, but, a fortiori, with probability one.

To prove the converse, let $\alpha_{n}$ denote the distribution function of the random variable $\zeta_{n}=\left(I_{1}+\cdots+I_{n}\right) / n$ and assume that for some constant random variable $c$, we have

$$
\lim _{n \rightarrow \infty} P\left[\left|\zeta_{n}-c\right| \geqq \epsilon\right]=0 \quad \text { for every } \epsilon>0 .
$$

Then

$$
\lim _{n \rightarrow \infty} P\left[c-\epsilon \leqq \zeta_{n} \leqq c+\epsilon\right]=1
$$

so that

$$
\lim _{n \rightarrow \infty} P\left[\zeta_{n} \leqq t\right]= \begin{cases}0 & \text { if } t<c, \\ 1 & \text { if } t \geqq c\end{cases}
$$


This shows that

$$
\lim _{n \rightarrow \infty} \alpha_{n}(t)= \begin{cases}0 & \text { if } t<c, \\ 1 & \text { if } t \geqq c .\end{cases}
$$

Now let $\alpha$ be the distributor of the sequence $\mu_{0}, \mu_{1}, \cdots$. As proved in Feller [1, p. 223],

$$
\lim _{n \rightarrow \infty} \alpha_{n}(t)=\alpha(t)
$$

at each point $t$ of continuity of $\alpha$. As a nondecreasing function, $\alpha$ has at most countably many points of discontinuity. Letting $\rho$ be an arbitrarily small positive number, we can therefore find points $p_{1}$ and $p_{2}$ of continuity of $\alpha$ such that $c-\rho<p_{1}<c<p_{2}<c+\rho$. It follows from (2) and our normalization agreement for distributors that

$$
\alpha(t)=\left\{\begin{array}{cl}
0 & \text { if } t<c, \\
\frac{1}{2} & \text { if } t=c, \\
1 & \text { if } t>c .
\end{array}\right.
$$

Thus, from the representation

$$
\mu_{n}=\int_{0}^{1} t^{n} d \alpha(t)
$$

we obtain $\mu_{n}=c^{n}$. But this means that if $m$ is any positive integer and $n_{1}<\cdots<n_{m}$ any $m$ indices, then

$$
P\left(A_{n_{1}} \cdots A_{n_{m}}\right)=P\left(A_{n_{1}}\right) \cdots P\left(A_{n_{m}}\right) .
$$

Therefore, the sets $A_{1}, A_{2}, \cdots$, and consequently, the corresponding indicators $I_{1}, I_{2}, \cdots$, are independent.

Theorem 3. Suppose $\mu_{0}, \mu_{1}, \cdots$ is admissible with respect to a probability space $(\Omega, A, P)$, sets $A_{n}$ in $A$, and distributor $\alpha$. Then the $A_{n}$ satisfy the converse of the Borel-Cantelli Lemma (as in Tucker [3, p. 70] ) if and only if $\alpha(0+)=0$. In fact, $P\left[A_{n}\right.$ i.o. $]=1-\alpha(0+)$.

Proof. Since

$$
P\left(\bigcap_{j=m}^{n} A_{j}^{c}\right)=\int_{0}^{1}(1-t)^{n-m+1} d \alpha(t)
$$

we obtain 


$$
\lim _{n \rightarrow \infty} P\left(\bigcap_{j=m}^{n} A_{j}^{c}\right)=\alpha(0+) .
$$

Now,

$$
\begin{aligned}
P\left[A_{n} \text { i.o. }\right] & =P\left(\bigcap_{m=1}^{\infty} \bigcup_{j=m}^{\infty} A_{j}\right) \\
& =\lim _{m \rightarrow \infty} P\left(\bigcup_{j=m}^{\infty} A_{j}\right)=1-\lim _{m \rightarrow \infty} P\left(\bigcap_{j=m}^{\infty} A_{j}^{c}\right) \\
& =1-\lim _{m \rightarrow \infty} \lim _{n \rightarrow \infty} P\left(\bigcap_{j=m}^{n} A_{j}^{c}\right) \\
& =1-\lim _{m \rightarrow \infty} \alpha(0+)=1-\alpha(0+) .
\end{aligned}
$$

THEOREM 4. Suppose $\mu_{0}, \mu_{1}, \cdots$ is admissible with respect to sets $A_{1}, A_{2}, \cdots$ and distributor $\alpha$. Then

$$
P\left[\bigcup_{n=1}^{\infty} A_{n}\right]=P\left[A_{n} \text { i.o. }\right] .
$$

Thus, with probability one, if a point lies in any $A_{n}$, then it lies in infinitely many $A_{n}$ 's.

Proof.

$$
\begin{aligned}
P\left[\bigcup_{n=1}^{\infty} A_{n}\right] & =P\left(A_{1}\right)+P\left(A_{2}-A_{1}\right)+P\left(A_{3}-\left(A_{2} \cup A_{1}\right)\right)+\cdots \\
& =P\left(A_{1}\right)+P\left(A_{2} A_{1} c\right)+P\left(A_{3} A_{2}{ }^{c} A_{1}{ }^{c}\right)+\cdots \\
& =\sum_{n=1}^{\infty}(-1)^{n-1} \Delta^{n-1} \mu_{1}
\end{aligned}
$$

by de Finetti's theorem

$$
=1-\alpha(0+)
$$

by Hardy's theorem

$$
=P\left[A_{n} \text { i.o. }\right]
$$

by Theorem 3 .

THEOREM 5. If $P\left(\bigcup_{n=1}^{\infty} A_{n}\right)=1$ and $P\left(\bigcap_{n=1}^{\infty} A_{n}\right)=0$, or, equivalently, if $\alpha(0+)=0$ and $\alpha(1-)=1$, then with probability one, a point 
of $\Omega$ lies in infinitely many $A_{n}$ 's and in infinitely many $A_{n}{ }^{c} s$.

Proof. If

$$
P\left(\bigcap_{n=1}^{\infty} A_{n}\right)=0
$$

then

$$
P\left(\bigcup_{n=1}^{\infty} A_{n}{ }^{c}\right)=1
$$

By Theorem 4

$$
P\left[A_{n}{ }^{c} \text { i.o. }\right]=1 \text {. }
$$

Also by Theorem 4

$$
P\left[A_{n} \text { i.o }\right]=1,
$$

since

$$
P\left(\bigcup_{n=1}^{\infty} A_{n}\right)=1
$$

THEOREM 6. Suppose $\mu_{0}, \mu_{1}, \cdots$ is a minimally completely monotonic sequence with $\mu_{1}<1$, with respect to sets $A_{1}, A_{2}, \cdots$. Then for every finite collection $A_{i_{n}}, \cdots, A_{i_{n}}, P\left(\bigcup_{j=1}^{n} A_{i j}\right)<1$, while for every infinite collection $A_{i_{1}}, A_{i_{2}}, \cdots, P\left(\bigcup_{j=1}^{\infty} A_{i_{j}}\right)=1$. That is, no finite collection of $A_{i}$ 's covers $\Omega$, while every infinite collection does cover $\Omega$, with probability one.

Proof.

$$
\begin{aligned}
P\left[\bigcup_{j=1}^{n} A_{i j}\right] & =\sum_{i=0}^{n-1}(-1)^{i} \Delta^{i} \mu_{1} \\
& <\sum_{i=0}^{\infty}(-1)^{i} \Delta^{i} \mu_{1}=\mu_{0}=1,
\end{aligned}
$$

while

$$
P\left(\bigcup_{j=1}^{\infty} A_{i_{j}}\right)=\sum_{i=0}^{\infty}(-1)^{i} \Delta^{i} \mu_{1}=1
$$

by Hardy's theorem. 


\section{REFERENCES}

1. W. Feller, An introduction to probability theory and its applications. Vol. II, Wiley, New York, 1966. MR 35 \#1048.

2. G. H. Hardy, Divergent series, Clarendon Press, Oxford, 1949. MR 11, 25.

3. H. G. Tucker, A graduate course in probability, Probability and Math Statist., vol. 2, Academic Press, New York, 1967. MR 36 \#4593.

4. D. V. Widder, The Laplace transform, Princeton Math. Series, vol. 6 Princeton Univ. Press, Princeton, N. J., 1946. MR 3, 232.

University of Evansville, Evansville, Indiana 47701 